\title{
Turner Syndrome and Inflammatory Bowel Disease:
}

\section{Case Report}

Priscilla Pereira dos Reis ${ }^{1}$, Ricardo Fortes Monteiro de Castro ${ }^{2}$, Rafaela Costa Vieira ${ }^{3}$ and Carolina de Paula Guimarães Baía ${ }^{3}$

1. Department of Gastroenterology and Hepatology, Felicio Rocho Hospital, Minas Gerais 30110-017, Brazil

2. Specialization in Gastroenterology and Hepatology, Felicio Rocho Hospital, Minas Gerais 30110-017, Brazil

3. Department of Gastroenterology and Hepatology; Inflammatory Bowel Disease Outpatient Clinic, Felicio Rocho Hospital, Minas Gerais 30110-017, Brazil

\begin{abstract}
Summary: TS (Turner syndrome) is a genetic disease which affects women caused by complete or partial absence of one of the $\mathrm{X}$ chromosomes. This condition is related to an increase in autoimmune diseases incidence like, IBD (inflammatory bowel disease). Material and Methods: a case report of a 31 years old woman with TS hospitalized with two months of prostration, fever, rashes, nausea, vomit and diarrhea. Investigations have shown colonic mucosa inflammation, besides chronic colitis with cryptic microabscess in the anatomopathological evaluation, suggesting IBD as etiology. Treatment with mesalazine was effective in symptoms improvement. Conclusions: TS is a condition frequently associated with autoimmune diseases, including diseases of the gastrointestinal tract, such as IBDs. Recognition and early treatment of this condition leads to an improvement in the quality of life of these patients and reduces the chances of future complications.
\end{abstract}

Key words: TS, inflammatory bowel diseases, colitis (ulcerative).

\section{Introduction}

TS (Turner syndrome) is a complex genetic anomaly which affects women due to total or partial absence of a sex chromosome that most often relates to $\mathrm{XO}$ karyotype in a feminine phenotype [1]. It affects 50:1,000,000 live born. The high susceptibility to autoimmunity in TS patients is well established, with an increase in the incidence of diseases such as type 1 diabetes, hypothyroidism, celiac disease and IBD (inflammatory bowel disease) [2, 3].

IBD affects millions of people around the world and the peak of incidence occurs between 15 and 30 years old [4]. Two chronic disorders represent this group of diseases: CD (Crohn's disease) and UC (ulcerative colitis) [5]. It is unclear why autoimmune diseases have an increased incidence in TS patients [6], but hormone therapy often used in these patients, seems to be a

Corresponding author: Priscilla Pereira dos Reis, M.D., research fields: gastroenterology and hepatology. susceptibility factor to IBD occurrence [7].

\section{Case Report}

KMA, female, 31 years old, carrier of TS, with a previous morbid history of cholecystitis, prolapsed of the mitral valve and obstructive sleep apnea. She was referred to our clinical center with a report of prostration, fever, skin rash, nausea and vomits, watery and mucoid diarrhea and abdominal discomfort, especially after feeding on breads and dairy products, with two months of evolution. She was treated in this period with nitazoxanide (considering that intestinal parasitoses are prevalent in our environment) plus prebiotics and racecadotril, in addition to a gluten-free diet, without any improvement. At the admission she performed laboratory tests, that evidenced: $\mathrm{Hb}, 9.2$ g/dL; Ht, 27.4\%; WBC, 8.000/uL; AST, 14 U/L; ALT, 29 U/L; GGT: 485 U/L; ALP:196 U/L; CRP, 35 mg/dL and ESR (erythrocyte sedimentation rate) of $33 \mathrm{~mm} / \mathrm{h}$. Abdominal tomography performed at the same time 
showed mild hepatosplenomegaly, gallbladder stones, discreet mesenteric and retroperitoneal lymphadenomegaly, diffuse thickening of the colon walls, without distension and small amount of free fluid in the peritoneal cavity. MR Cholangiography showed a $10 \times 8 \mathrm{~mm}$ gallbladder stone with no signs of obstruction or cholangitis.

With these findings we requested EGD (esophagogastroduodenoscopy) and colonoscopy thinking about the diagnosis of inflammatory bowel disease. EGD found two small ulcers covered by exudate in the distal esophagus, with signs of mild chronic pangastritis in the biopsies that also searched for Helicobacter pylori and it was negative. Colonoscopy showed diffuse enanthem, mainly in the ascending colon and cecum. The sigmoid and rectal mucosa was slightly swollen and friable, with ileocecal valve and terminal ileum preserved. Biopsies of the colon segments described cryptic microabscesses and infiltrate of mononuclear and neutrophils in the lamina propria. All these findings were compatible with chronic colitis, which could correspond with inflammatory bowel disease.

Considering the continuous pattern of involvement of all the colonic mucosa, absence of signs of stenosis to the colonoscopy, in addition to the highly suggestive biopsy, we started treatment with mesalazine $4 \mathrm{~g}$ /day. Patient presented fast and satisfactory improvement of the evacuation pattern, abdominal discomfort and her general condition. The treatment was well tolerated without any side effects at the first week of treatment, with hospital discharge for ambulatorial follow-up.

\section{Discussion}

TS is the most common chromosomal abnormality in women [6]. These patients have a higher risk to develop autoimmune disease, including those with a higher frequency in men [7]. Table 1 shows the incidence of autoimmune diseases in a European cohort. The relationship between TS and IBD has been described for over 40 years $[8,9]$. The incidence of $C D$ and UC in these patients is same, each one is responsible for $50 \%$ of IBD's cases [10]. After colonoscopy and biopsy, the main hypothesis for our patient was UC. Although this theme has been currently quite studied the true influence of autoimmunity in those patients remains unclear [11], as well as the physiopathology of this association $[12,13]$.

Table 1 Incidence of autoimmune diseases in two European populations with TS [14, 15].

\begin{tabular}{lllll}
\hline & \multicolumn{3}{c}{ English Population with TS $(\mathrm{N}=2,459)$} & Danish Population with TS (N=798) \\
\cline { 2 - 5 } & 4 & OC IR $(95 \%$ CI $)$ & OC IR (95\% CI) \\
\hline Addison's disease & 2 & $8.8(2.4$ to 22.6$)$ & - & - \\
Alopecia areata & $40.4(2.5$ to 75.0$)$ & - & $3.7(0.2-16.4)$ \\
Ankylosing spondylitis & 4 & $5.6(1.5$ to 14.4$)$ & 1 & $2.7(0.2-11.7)$ \\
Celiac disease & 45 & $14.0(10.2$ to 18.8$)$ & 1 & $1.5(0.4-3.9)$ \\
Crohn's disease & 27 & $5.3(3.5$ to 7.8$)$ & 3 & $4.1(2.5-6.3)$ \\
Diabetes mellitus, type 1 & 72 & $8.2(6.4$ to 10.3$)$ & 18 & $7.0(2.2-16.2)$ \\
Dupuytren's contracture & 7 & $9.6(3.9$ to 19.9$)$ & 4 & - \\
Halo nevus & 2 & $8.7(1.0$ to 31.5$)$ & - & $14.6(6.7-27.1)$ \\
Hashimoto's thyroiditis & 9 & $12.5(5.7$ to 23.9$)$ & 8 & - \\
Hyperthyroidism & 27 & $4.9(3.2$ to 7.1$)$ & - & - \\
Hypothyroidism & 293 & $8.8(7.8$ to 9.9$)$ & - & - \\
Pernicious anaemia & 6 & $3.3(1.2$ to 7.2$)$ & - & $1.6(0.4-4.2)$ \\
Psoriasis & 26 & $5.4(3.5$ to 8.0$)$ & 3 & $0.9(0.2-2.4)$ \\
Rheumatoid arthritis & 23 & $1.8(1.2$ to 2.7$)$ & 3 & $2.5(1.2-4.7)$ \\
Ulcerative colitis & 18 & $3.9(2.3$ to 6.1$)$ & 8 & \\
OC = Observed cases & & & & \\
IR = Incidence ratio & & & & \\
\hline
\end{tabular}


In the presented case, the patient had no diagnosis of autoimmune disease until hospitalization and the gluten-free diet, besides being very restrictive, had not led to symptoms relief. Celiac disease was not tested before diet restriction.

Symptoms related to gastrointestinal diseases in ST patients usually occur at young age, around 16 years old [16]. Young people with IBD have a higher risk of developmental delay and growth retardation, especially during disease activity $[17,18]$. Our patient opened the intestinal tract later, at age 31 . For this reason, early screening for autoimmune diseases in these patients should be routine in order to identify and treat them in a timely manner [19]. Intestinal parasitosis in young age also leads to developmental delay if not recognized and treated. In low-income communities it is very common use prophylactic treatment for parasitosis. In our case this management was performed with no success.

Some hypotheses for the relationship between TS and autoimmune diseases have been proposed during in recent years. Recently, 7 cases of TS patients who developed CD after hormone replacement have been described in Japan [11]; Meneghelli described a case of a patient who started CD's symptoms right after puberty [20], both suggesting relationship between hormonal changes and autoimmunity.

It is well known that immunogenicity is not increased in TS, so it is postulated that abnormalities related to the $\mathrm{X}$ chromosome and the genes found in it may be involved in the immune response [21,22].

Autoimmune diseases are known to be more common in women. In those with TS and an isochromosome $\mathrm{X}$ there is an increase in the incidence of IBD, maybe due to the possibility that the immune dysfunction is associated to a gene of the long arm of this chromosome [22]. The karyotype of our patient was not known by her or her relatives and it was not tested during her hospitalization period.

Aminosalicylates are the medication of choice to start treatment in UC cases [23, 24]; and this patient showed improvement with mesalazine in high dose (4 g per day), which was well tolerated, leading to great reduction in diarrhea and abdominal pain. In this case the presence of ST did not seem to influence in the natural course of the disease.

The goals of IBD treatment include not only symptoms relief, but also improvement in quality of life, deep remission and improvement of endoscopic mucosa aspect and histological healing [25]. UC cases without treatment or with inadequate treatment may evolve with some complications like need of colectomy or colon tumors [26, 27]. The follow-up of these patients should be regular and the medication maintained in the long term [28].

\section{Conclusions}

TS is a disease frequently associated with autoimmunity, in this specific case, to IBD, which may cause functional limitation and profound psychological impact. Screening for autoimmune diseases in this group of patients provides early diagnosis and treatment, avoiding developmental delay and future complications. In the case of UC, specific therapy is indicated for the diagnosis of symptoms, endoscopic and histological healing of the mucosa and prevention of future complications, such as colorectal cancer.

\section{Acknowledgments}

We thank dear Ana Luíza who gently reviewed this English version.

\section{Declaration of Interest}

The authors report no conflicts of interest.

\section{References}

[1] Maffè, G. C., Calcaterra, V., Toglia, R., Formagnana, P., Miceli, E., Corazza, G. R., and Larizza. D. 2013. "Usefulness of Abdominal Ultrasonography with Studies of the Intestinal Loops in Turner Syndrome Patients." Journal of Ultrasound 16 (3): 97-100.

[2] Gravholt, C. H. 2005. "Clinical Practice in Turner Syndrome." Nature Clinical Practice Endocrinology \& Metabolism 1: 41-52.

[3] Mortensen, K. H, Cleemann, L., Hjerrild, B. E., Nexo, E., Locht, H., and Jeppesen, E. M. 2009. "Increased 
Prevalence of Autoimmunity in Turner Syndrome-Influence of Age." Clinical and Experimental Immunology 156: 205-10. 2009

[4] Loftus, E. V. J., and Sandborn, W. J. 2002. "Epidemiology of Inflammatory Bowel Disease." Gastroenterology Clinics of North America. 31 (1): 1-20.

[5] Clara, A., and Cho, J. H. 2009. "Inflammatory Bowel Disease." The New England Journal of Medicine 361 (21): 2066-78.

[6] Elshieikh, M., Conway, G. S., and Wass, J. A. 1999. "Medical Problems in Adult Women with Turner's Syndrome." Annals of Medicine 31 (2): 99-105.

[7] JorgensenK. T., et al. 2010. "Autoimmune diseases in women with Turner's syndrome." Arthritis and Rheumatology. 62658-666.

[8] Price, W. H. 1979. "A High Incidence of Chronic Inflammatory Bowel Disease in Patients with Turner's Syndrome." Journal of Medical Genetics 16 (4): 263-6.

[9] Weinrieb, I. J., Fineman, R. M., and Spiro, H. M. 1976. "Turner Syndrome and Inflammatory Bowel Disease." New England Journal of Medicine. 294: 1221-2.

[10] Manzione, N. C.et al. 1988. "Turner's Syndrome and Inflammatory Bowel Disease: A Case Report with Immunologic Studies." American Journal of Gastroenterology 83 (11): 1294-7.

[11] Bakalov, V. K., Kram, M., Kram, E., and Das, K. M. 2012. "Autoimmune Disorders in Women with Turner Syndrome and Women with Karyotypically Normal Primary Ovarian Insufficiency.” Journal of Autoimmunity 38 (4): 315-21.

[12] Ihara, Y., Hizawa, K., Fujita, K., Iida, M., Washio, E., Kai, T., Nitahata, T., Esaki, M., and Iida, M. 2017. "A Case of Turner Syndrome (46XXp-/45X) Complicated with Crohn's Disease after Hormone Therapy." The Japanese Journal of Gastro-Enterology 114 (3): 445-9.

[13] Lleo, A., Moroni, L., Caliari, L., and Invernizzi, P. 2012. "Autoimmunity and Turner's Syndrome." Autoimmunity Reviews 11 (6-7): A538-43.

[14] Jorgensen, K. T., Rostgaard, K., Bache, I., Biggar, R. J., Nielsen, N. M., Tommerup, N., and Frisch, M. 2010. "Autoimmune Diseases in Women with Turner's Syndrome." Arthritis \& Rheumatism 62: 658-66. doi:10.1002/art.27270

[15] Goldacre, M. J., and Seminog, O. O. 2014. "Turner Syndrome and Autoimmune Diseases: Record-Linkage Study." Archives of Disease in Childhood 99: 71-3.

[16] Takaya, J., Teraguchi, M., Ikemoto, Y., Yoshimura, K., Yamato, F., Higashino, H., Kobayashi, Y., and Kaneko, K. 2006. "Turner Syndrome Associated with Ulcerative Colitis." Clinical Pediatric Endocrinology 15 (3):
97-100.

[17] Motil. K. J., Grand, R. J., Davis-Kraft, L., Ferlic, L. L., and Smith, E. O. 1993. "Growth Failure in Children with Inflammatory Bowel Disease: A Prospective Study." Gastroenterology 105 (3): 681-91.

[18] Durusu, M., Gürlek, A., Şimşek, H., Balaban, Y. H., and Tatar, G. 2005. "Coincidence or Causality: Celiac and Crohn Disease in a Case of Turner Syndrome." The American Journal of the Medical Sciences 329 (4): 214-6.

[19] Larizza, D., Calcaterra, V., and Martinetti, M. 2009. "Autoimmune Stigmata in Turner Syndrome: When Lacks an X Chromosome.” Journal of Autoimmunity. 33 (1): 25-30.

[20] Meneghelle, U. G. 1983. "Crohn Disease Associated with Turner's Syndrome (a possible Physiopathological Mechanism)." Archives of Gastroenterology 20 (1): 17-9.

[21] Hayward, P. A., Satsangi, J., and Jewell, D. P. 1996. "Inflammatory Bowel Disease and the X Chromosome." Quarterly Journal of Medicine 89 (9): 713-8.

[22] Hyodo, H., Tomita, Y., Hirai, K., Hirakawa, H., Ueno, S., and Ishiguro, H. 2009. "Turner Syndrome with Ulcerative Colitis." Clinical Pediatric Endocrinology 18 (4): 101-5.

[23] Haus, O., Martinsen, T. C., and Waldum, H. 2015. "5-Aminosalicylic Acid, a Specific Drug for Ulcerative Colitis." Scandinavian Journal of Gastroenterology 50 (8): 933-41.

[24] Probert C. S., Dignass, A. U., Lindgren, S., Oudkerk Pool, M., and Marteau, P. 2014. "Combined Oral and Rectal Mesalazine for the Treatment of Mild-to-Moderataly Active Ulcerative Colitis: Rapid Symptom Resolution and Improvements in Quality of Life." Journal of Crohn and Colitis 8 (3): 200-7.

[25] Feuerstein, J. D., and Cheifetz, A. S. 2014. "Ulcerative Colitis: Epidemiology, Diagnosis, and Management." Mayo Clinic Proceedings 89 (11): 1553-63.

[26] Klotz, C., Barret, M., Dhooge, M., Oudjit, A., Chaussade, S., Coriat, R., and Abitbol, V. 2015. "Management of Diagnosis and Treatment in Ulcerative Colitis." Press Med 44 (2): 144-9.

[27] Silva, B. C., Lyra, A. C., Rocha, R., and Santana, G. O. 2014. "Epidemiology, Demographic Characteristics and Prognostic Predictors of Ulcerative Colitis." World Journal of Gastroenterology 20 (28): 9458-67.

[28] Bressler B., Marshall, J. K., Bernstein, C. N., Bitton, A., Jones, J., Leontiadis, G. I., Panaccione, R., Steinhart, A. H., Tse, F., and Feagan, B. 2015. "Clinical Practice Guidelines for the Medical Management of Nonhospitalized Ulcerative Colitis: The Toronto Consensus.” Gastroenterology 148 (5): 1035-58. 